\title{
WIDE FIELD CAMERA OBSERVATIONS OF BAADE'S WINDOW ${ }^{1}$
}

\author{
JoN A. HolTZMAN \\ Lowell Observatory, 1400 West Mars Hill Rd., Flagstaff, Arizona 86001 \\ Electronic mail: holtz@lowell.edu \\ R. M. LIGHT \\ Infrared Processing and Analysis Center, 100-22, California Institute of Technology, Pasadena, California 91125 \\ Electronic mail: light@ipac.caltech.edu
}

\section{WILLIAM A. BAUM}

Astronomy Department, University of Washington, FM-20, Seattle, Washington 98195

Electronic mail: baum@ astro.washington.edu

\section{GUY WORTHEY}

Department of Astronomy, University of Michigan, Ann Arbor, Michigan 48109 Electronic mail: worthy@astro.lsa.umich.edu

\section{S. M. FABER}

UCO/Lick Observatories, Board of Studies in Astronomy and Astrophysics, University of California, Santa Cruz, Santa Cruz, California 95064

Electronic mail: faber@lick.ucsc.edu

\section{DEIDRE A. HUNTER}

Lowell Observatory, 1400 West Mars Hill Rd., Flagstaff, Arizona 86001

Electronic mail: dah@ lowell.edu

EARL J. O'NeIL, JR.

Kitt Peak National Observatory, National Optical Astronomy Observatories, ${ }^{2}$ P.O. Box 26732, Tucson, Arizona 85726 Electronic mail: oneil@ noao.edu

\section{TOBIAS J. KREIDL}

Lowell Observatory, 1400 West Mars Hill Rd., Flagstaff, Arizona 86001 Electronic mail: tjk@lowell.edu

\section{E. J. GROTH}

Physics Department, Princeton University, Box 708, Princeton, New Jersey 08544 Electronic mail: groth @ pupgg.princeton.edu

\section{JAMES A. WESTPHAL}

Division of Geological and Planetary Sciences, 170-25, California Institute of Technology, Pasadena, California 91125 Electronic mail: jaw @ soll.gps.caltech.edu

Received 1992 November 9; revised 1993 July 8

\begin{abstract}
We have observed a field in Baade's Window using the Wide Field Camera of the Hubble Space Telescope (HST) and obtain $V$ - and $I$-band photometry down to $V \sim 22.5$. These data go several magnitudes fainter than previously obtained from the ground. The location of the break in the luminosity function suggests that there are a significant number of intermediate age $(<10 \mathrm{Gyr})$ stars in the Galactic bulge. This conclusion rests on the assumptions that the extinction towards our field is similar to that seen in other parts of Baade's Window, that the distance to the bulge is approximately $8 \mathrm{kpc}$, and that we can determine fairly accurate zero points for the $H S T$ photometry. Changes in any one of these assumptions could increase the inferred age, but a conspiracy of lower reddening, a shorter distance to the bulge, and/or photometric zero-point errors would be needed to imply a population entirely older than $10 \mathrm{Gyr}$. We infer an initial mass function slope for the main-sequence stars, and find that it is consistent with that measured in the solar neighborhood; unfortunately, the slope is poorly constrained because we sample only a narrow range of stellar mass and because of uncertainties in the observed luminosity function at the faint end.
\end{abstract}




\section{INTRODUCTION}

Understanding the stellar population in the Galactic bulge is important for several reasons. Stars in the bulge have different physical properties (e.g., metallicity) compared with stars in the solar neighborhood, and the conditions under which they formed may have been different from those for nearby stars. To produce a star formation history for our Galaxy requires a knowledge of the ages and compositions of the bulge stars. The star formation history of the Galactic bulge may provide important clues towards understanding the formation of other spheroidal systems; in any case, an understanding of Galactic bulge stars is necessary if we are to model bulge systems of other galaxies, in which we cannot resolve individual stars.

Baade (1946,1951) identified several "windows" of moderately low obscuration in the direction of the Galactic bulge. One of the most well studied is known as Baade's Window and is located at a Galactic latitude of $b=3.9^{\circ}$, centered on the globular cluster NGC 6522. The partially clear region is about a degree across, with various amounts of extinction. The Baade's Window field is crowded, and accurate photometry is difficult.

The brightest stars in Baade's Window, $\mathbf{M}$ and $\mathrm{K}$ giants, have been studied spectroscopically by a number of authors (Whitford \& Rich 1983; Rich 1988; Terndrup et al. 1991). These observations show that a significant number of bulge giants are metal rich. There is a wide range of metalliticies, however, ranging approximately from $1 / 10$ to 10 times solar. The mean metallicity appears to be about twice solar (Rich 1988).

Previous photometric work has been limited by the crowding in the field. The deepest survey is that of Terndrup (1988), who sampled a field in Baade's Window as well as several other fields at higher Galactic latitude. In Baade's Window, Terndrup was able to observe down to $V \sim 20$, which is near the main-sequence turnoff of the bulge population. Both a bulge giant branch and a foreground main sequence can be seen in Terndrup's colormagnitude diagram. Terndrup detected a metallicity gradient in the bulge from the differing locations of the giant branches in his fields. Geisler \& Friel (1992) used Washington band photometry to determine metallicities of $G$ and $\mathrm{K}$ giants in Baade's Window and found results similar to those obtained spectroscopically, namely a wide range of metallities and a mean $[\mathrm{Fe} / \mathrm{H}]$ of 0.17 dex.

There has been little direct measurement of the age of the bulge population. Terndrup concluded that all of the bulge stars had ages consistent with those of the globular clusters, between 10 and 15 billion years. However, his conclusion with regard to Baade's Window was somewhat uncertain since he did not have data fainter than the turnoff. Also, he adopted a distance to the bulge of $7 \mathrm{kpc}$ and used isochrones appropriate for stars with less than solar

\footnotetext{
${ }^{1}$ Based on observations with the NASA/ESA Hubble Space Telescope, obtained at the Space Telescope Science Institute, which is operated by AURA, Inc., under NASA contract NAS 5-26555.

${ }^{2}$ Operated by the Association of Universities for Research in Astronomy, Inc., under cooperative agreement with the National Science Foundation.
}

metallicity; for larger values of either distance or metallicity, the inferred age would be smaller.

Other estimates of the age have been made from observations of luminous late-M giants. From pulsation, Wood \& Bessell (1983) inferred ages of only a few Gyrs for some of the bulge giants. Periods of Miras in the bulge are longer than those of globular cluster giants, implying larger luminosities and smaller ages, although this conclusion is tentative because of uncertainties in Mira evolution, especially for metal-rich stars. The presence of RR Lyraes in the bulge implies that there are some stars that are at least 10 Gyr old; from an analysis of the metallicity distribution of the RR Lyraes, Lee (1993) suggests that the bulge RR Lyraes are older than those in the halo and infers that the bulge may have been the first part of our Galaxy to form. Consequently, there is no strong agreement on the age of the Galactic bulge; Rich (1992) presents a good review of the information relevant to age determination of bulge stars.

We have observed a Baade's Window field with the Hubble Space Telescope to use its higher resolution to reduce the crowding. Our main goal was to resolve stars past the turnoff and to determine the nature of the bulge population. In particular, we were interested in determining the luminosity function of the main-sequence stars to find out whether the initial mass function (IMF) in the metalrich bulge differs from that in other regions. The study of main-sequence stars avoids many of the uncertainties in the evolution of metal-rich stars onto the giant branch; also, the small range in stellar mass available on the giant branch leads to a large uncertainty in the IMF.

\section{OBSERVATIONS}

A Baade's Window field was observed with the Wide Field Camera on 1991 August 18 using the F555W and F785LP filters. In F555W, two exposures of $800 \mathrm{~s}$ and two of $1000 \mathrm{~s}$ were taken. In F785LP, four exposures of $1000 \mathrm{~s}$ were obtained. The Wide Field Camera (WFC) field lies in the northern section of Baade's Window, at $\alpha=18: 03: 10.0$, $\delta=-29: 51: 43$ (2000). This location was chosen because it appears to be the least obscured region judging from a visual inspection of POSS prints.

In addition to the Baade's Window frames, observations were taken of the Galactic open cluster NGC 188, which provides a sparse field of stars used to construct point spread functions (PSFs) for the crowded field photometry in the Baade's Window frames. In NGC 188, frames were taken at four overlapping pointings to provide full field coverage for the PSF. In practice, however, several of these pointings either did not contain enough stars or had large amounts of jitter. Consequently, for use in PSF construction, we have used just one of the pointings in NGC 188; all of the pointings were used, however, to estimate the error in photometric zero points arising from PSF variability, as discussed below.

Our images were processed using the techniques discussed by Lauer (1989), employing calibration data obtained near in time to the observations. An A-to-D correc- 
tion was applied, and a bias level was subtracted using the information from the overscan region. Preflash, bias structure, and dark frames were subtracted, and the data were flattened with flat fields constructed from several sweeps across the bright earth. The flat fields for F555W had to be taken with a neutral density filter. These flat fields are of sufficiently high $\mathrm{S} / \mathrm{N}$ to accurately correct for pixel-topixel variations in detector response, but are less accurate for large scale flat field errors (WFPC Science Verification report 1992). As discussed below, we used the NGC 188 observations, for which ground-based $V$ magnitudes are available, to quantify the flat-field errors and determined that the flats produce one-sigma scatter at a level of about five percent.

The four exposures in each filter were combined to produce a single averaged picture. During the averaging process, cosmic rays were removed by ignoring pixels that were statistically significantly larger than expected from the corresponding pixels on the other frames, using a technique developed by the WFPC IDT (WFPC SV report 1992); this procedure works extremely well when the individual frames are well registered. The final average frames were visually inspected to insure that the procedure did not affect the bright central pixels of stars.

\section{ANALYSIS}

The WFC image in F555W is shown in Fig. 1 (Plate 93). Clearly, the field is crowded. There are a significant number of saturated stars. The F785LP frame is deeper and contains even more saturated stars.

Accurate photometry in such a crowded field, especially for the fainter stars, requires accurate simultaneous PSF fitting of neighboring stars. Unfortunately, this is difficult for WFC frames because the PSF has large variations across the field of view, and can change slightly from exposure to exposure because of telescope jitter. The crowding of the Baade's Window field prevents a determination of the PSF from the frame itself, especially if one requires different PSFs for different regions of the chip.

Separate PSFs for each of the four chips were constructed from the NGC 188 frames. In these frames, there were only 3-6 well exposed stars per chip. We did not feel that there was sufficient information to attempt to construct a field-dependent PSF. Rather, we have constructed a single "average" PSF from the stars on each chip. The PSF representations were made using standard DAOPHOT (Stetson 1987) techniques, i.e., a single Gaussian aligned with pixels plus an empirical residual table. We made the PSF from the pointing in NGC 188 which provided the largest number of stars per chip.

We have also tried to reduce one of the frames using model field-dependent PSFs, where the models are simple diffraction models with optical parameters relevant for the HST (e.g., Burrows et al. 1991). The initial results were similar to those obtained using the NGC 188 PSFs; the scatter in the locus of points in a color-magnitude diagram was similar, although there was several hundredths of a magnitude more scatter with the model PSFs. With more effort at determining better model parameters, the model PSF results might be improved, but we think that it is unlikely that they will change the results qualitatively. We estimated the error introduced by using a field-independent PSF by PSF-fitting the NGC 188 frames and comparing the PSF fit results with the aperture results; we found that the PSF-fitting introduced scatter of about 6 percent. This comparison was made for all four pointings in NGC 188 (recall, the PSF was constructed from just one of these), so this procedure also tested the errors arising from a timevariable PSF. We used the NGC 188 frames to aperture correct the Baade's Window frame; since the combined four pointings in NGC 188 provided samples of stars over a reasonable fraction of the WF field-of-view and also included several different amounts of jitter, systematic errors arising from the use of an inaccurate PSF should have been largely removed.

Stars were automatically detected on the Baade's Window frames using the DAOPHOT FIND routine. To avoid saturated stars and spurious detections in the extended wings of these stars, all objects falling within 12 pixels of a saturated pixel were discarded. This resulted in throwing away about 15 percent of the area of the F555W frame, and 20 percent of the F785LP frame. A threshold of 15 counts (in the averaged frame) over the local background was used as a criterion for the star detection; since the background varied significantly over the field of view (presumably because of differing components of unresolved stars over the field), this threshold corresponded to different levels of sigma over the background depending on location, but in no case was anything less than a $4 \sigma$ detection accepted. The threshold was chosen as the lowest which did not produce an unacceptable number of spurious detections (judged visually). On each of the eight frames (four chips, each through F555W and F785LP), approximately 4000 objects were detected.

To perform the profile fitting, we used a nonstandard technique for grouping the stars which uses a consistent grouping scheme for all of the stars and also saves a considerable amount of computing time (Holtzman 1989). In each frame, each star was grouped along with other stars falling within 15 pixels. The brightnesses of the stars in the group were determined using profile-fitting techniques, solving for the positions of the stars and their relative brightnesses compared with the PSF representation, as well as fitting for the background level. From the set of resulting magnitudes, however, only the results for the central star were kept, then a new group was created for the next star. This creates many more groups than using the standard technique but the size of each group is kept to a manageable number (usually 10-30 stars per group). With the standard technique, which continues to extend groups from all the neighbors of a given star, one ends up with most of the stars in groups with more than 100 stars unless rather small critical separations are used (smaller than 10 pixels), resulting in different grouping criteria for different stars or for unreasonably small groups for all of the stars. To assess crowding errors, we rereduced the frame using a critical separation of 10 pixels rather than 15 . The resulting 
color-magnitude diagram had more scatter than the diagram made with the 15 pixel separation; there were no systematic offsets in either the color-magnitude diagram or in the resulting luminosity functions.

During the profile fitting, we found that many real stars were dropped by the DAOPHOT NSTAR algorithm because it was finding that the fits were uncertain; we believe this was caused by using a less-than-perfect PSF. We modified the routine to make it impossible to drop stars for this reason. This left us with some spurious objects, but generally these were later removed either because they were extremely faint or because no corresponding object was found on the frame in the other color.

Stars were found independently on the F555W and F785LP frames. The two lists were subsequently merged using a distance criterion. For each star on the F555W frame, the F785LP frame was searched to find the nearest star within one pixel after the average global shift between the frames was applied. Only objects detected in both frames were kept as true detections.

The frames were transformed to standard $V$ and $I$ (Cousins) using WFC observations of $\omega$ Cen. Zero points are rather difficult to determine for $H S T$, as discussed in the WFPC Science Verification Report (1992). We adopted the following procedure for getting standard magnitudes.

First, the PSFs were used to reduce the NGC 188 frames, which have a few well exposed stars that are well separated. Aperture corrections were determined for each of the PSFs by averaging the difference between the PSF-fit magnitudes and large aperture magnitudes for the stars. These aperture corrections were derived from all four pointings in NGC 188, even though the PSF was constructed from only one of these; this should reduce any systematic errors that might arise from a different PSF between the PSF frame and the Baade's Window frame, since the different NGC 188 pointings also sample different PSFs (the PSFs vary from frame to frame because of variable pointing jitter in $H S T$ ). These aperture corrections were applied to the Baade's Window measurements.

Next, a zero point was applied to each of the Baade's Window frames to bring the flight magnitudes onto the ground-based WFPC system as presented in Harris et al. (1991). The zero points from the WFPC have been determined using observations of $\omega \mathrm{Cen}$, as presented in the WFPC Science Verification Report. However, the zero points vary with time because contaminants accumulate on the CCD windows as time passes; periodically, these contaminants are removed by warming up the WFPC CCDs. The rate of contaminant buildup is monitored monthly in the WF2 camera and these data show that the rate is repeatable between "cleanups," and that each cleanup returns the throughput to approximately the same level. Because these data exist only for WF2, we have chosen only to use the photometry from that camera, as zero points are more uncertain for the other cameras. We used the uncontaminated zero point for WF2 as presented in the WFPC Science Verification Report (1992). Since our observations were taken about 45 days after a "cleanup" we applied a
TABLE 1. Photometric zero points and transformation coefficients. ${ }^{1}$

\begin{tabular}{lcccc}
\hline \hline Filter & Chip & Aperture correction $^{2}$ & Zero point $^{3}$ & Transformation $^{4}$ \\
\hline F555W & 2 & -1.59 & -2.066 & -0.044 \\
F785LP & 2 & -1.87 & -3.454 & 0.158 \\
\hline
\end{tabular}

${ }^{1} \mathrm{~F} 555 \mathrm{~W}$ is transformed to $V$, and F785LP to Cousins $I$. The transformation equation is: standard $\mathrm{mag}=-2.5 \times \log (\mathrm{cnts} / \mathrm{s})+25+$ apcor + zero + trans $\times(V-I)$.

${ }^{2}$ The aperture corrections were determined using the NGC 188 frames. ${ }^{3}$ The zero point includes the shift from the flight to the ground WFPC system, plus a correction for contamination in the WFPC at the time of the observations, plus the zero point from the transformation between the ground WFPC system and the standard system.

${ }^{4}$ The color term comes from the ground observations of Harris et al. (1991).

correction of $0.05 \mathrm{mag}$ in F555W and $0.01 \mathrm{mag}$ in F785LP to account for contamination at the time of the observations. From the scatter in the monthly monitors, we judge that these corrections give zero points which should be accurate to approximately $0.02 \mathrm{mag}$.

Finally, the magnitudes on the ground-based system were transformed to Cousins $V$ and $I$ using the ground observations of Harris et al. (1991) to determine a linear transformation between $\mathrm{F} 555 \mathrm{~W}$ and $V$ and between F785LP and $I$ as a function of $V-I$. The aperture corrections, zero-point corrections, and transformation color terms are shown in Table 1.

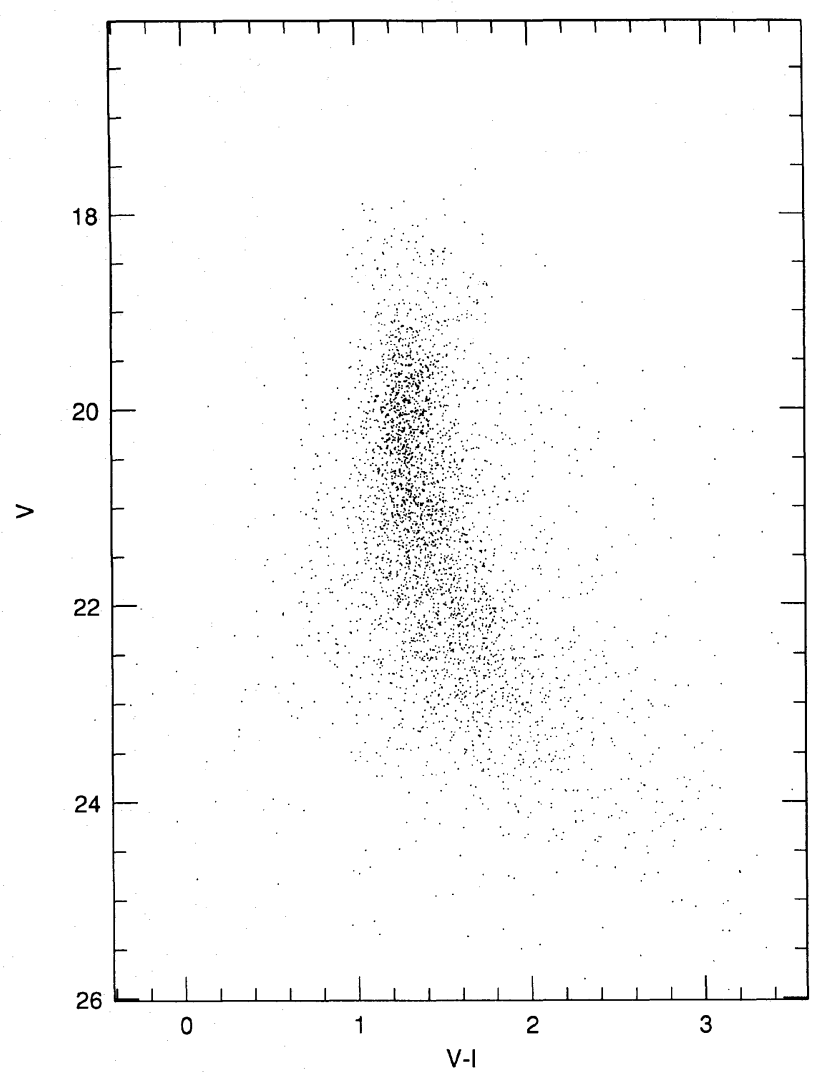

FIG. 2. Color-magnitude diagram for WF2. No correction for extinction or reddening has been applied. 
From the above procedure, we estimate that our zero point in each color should be good to better than $0.1 \mathrm{mag}$; the contamination correction is accurate to about 0.02 mag, the accuracy of the standard star measurements is several hundredths of a magnitude (WFPC SV report), and we estimate the accuracy in our aperture corrections to be a few hundredths of a magnitude judging from the scatter between the different pointings in NGC 188 . We checked the accuracy of the F555W zero point by comparing our photometry of NGC 188 with previously published $V$ photometry (Caputo et al. 1990). We found that our magnitudes agreed with those determined from the ground with a systematic offset of about $0.03 \mathrm{mag}$ (in the sense that the WFPC $V$ magnitudes were too faint). As a further check on our zero points, we obtained $V$ - and $I$-band frames in NGC 188 at Lowell Observatory with the 42" telescope and an $800 \times 800$ Loral CCD. The Lowell $V$ band data disagreed with the Caputo et al. data by about $0.08 \mathrm{mag}$. The disagreement was in the same direction as the disagreement of the WFPC data. With a larger number of stars overlapping between the WFPC frames and the Lowell frames, we found that the WFPC $V$ magnitudes were too bright as compared with the Lowell $V$ magnitudes by $0.03 \mathrm{mag}$; in the $I$ band, the scatter was larger and the WFPC magnitudes were brighter than the Lowell magnitudes by $0.06 \mathrm{mag}$. Although we are concerned with the discrepancy between the Lowell $V$ magnitudes and the Caputo et al. magnitudes (which are tied to previous work in the field), we were encouraged that by either comparison, our zero points seem to be accurate to better than 0.1 mag.

Random errors arise from a combination of photon statistics, uncertainties in the sky background, fitting and PSF uncertainties, uncertainties from confusion and unidentified stars, and flat fielding errors. Formal errors from photon statistics are small, less than a few percent even at $V \sim 22$. Background and confusion errors are difficult to estimate, but we believe that they could easily introduce scatter at the $\sigma=0.05$ to $0.1 \mathrm{mag}$ level. Fitting errors are returned by the PSF-fitting routine and are generally in the range of 0.05 to $0.1 \mathrm{mag}$. Finally, flat fielding errors were estimated to be approximately 0.05 to 0.10 mag judging from the scatter in the NGC 188 photometry when compared with previous ground-based photometry. These combine to give an estimated total random error per star on the order of $\sigma=0.15-0.2 \mathrm{mag}$. We note that since the bulge is an inhomogeneous sample of stars (in distance, metallicity, and probably age), an intrinsic scatter in photometry is expected, so we believe that random instrumental errors of $0.2 \mathrm{mag}$ do not significantly compromise our interpretation.

\subsection{Color-Magnitude Diagrams}

The color-magnitude diagram is presented in Fig. 2. No reddening or extinction correction has been applied. A main sequence is clearly discernible. Stars brighter than $V \sim 18-19$ (depending on pixel centering) are saturated, explaining the lack of bright stars and a giant branch in the diagram. At the faint end, we are almost complete down to $V \sim 21$, and become almost totally incomplete by $V \sim 23.5$. Completeness corrections are discussed below.

\subsection{Raw Luminosity Function}

A raw luminosity function for the F555W exposure is shown in Fig. 3, where we plot the number of stars on the

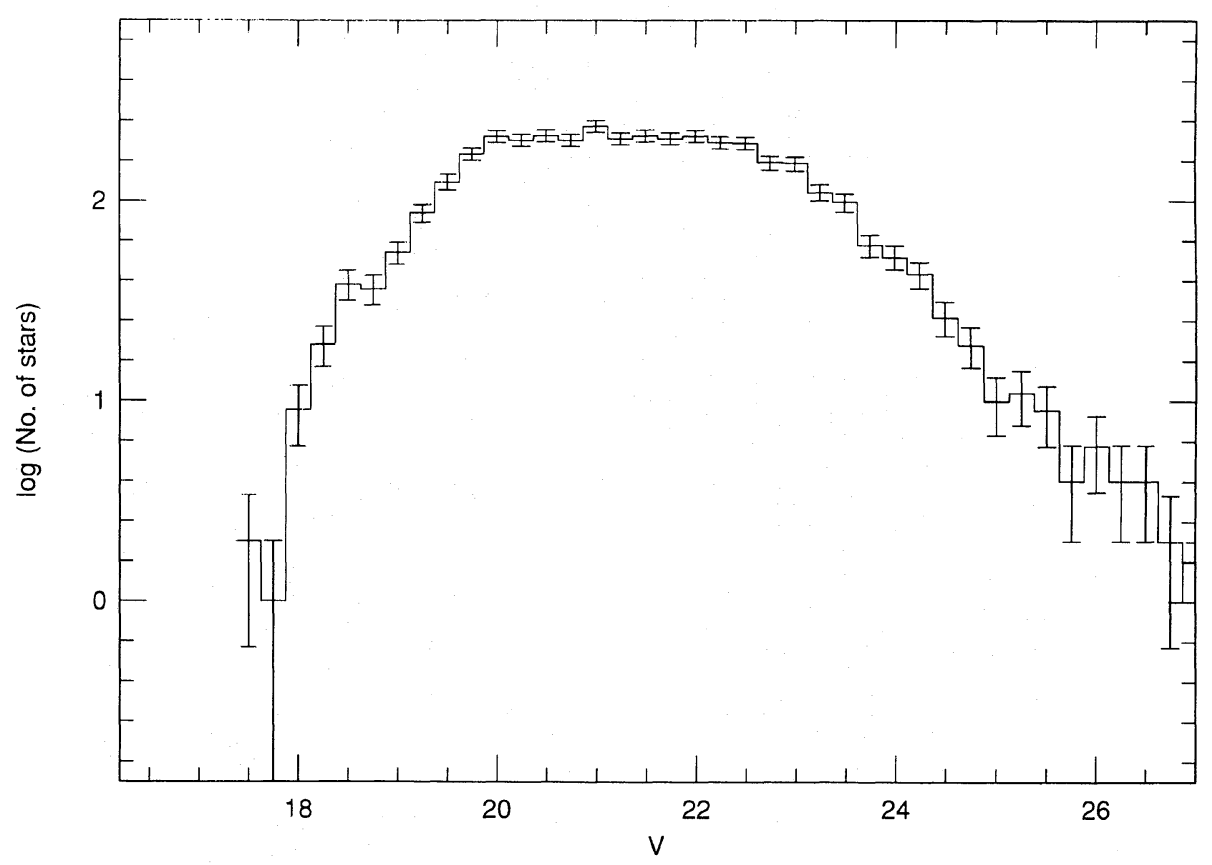

FIG. 3. Raw luminosity function for WF2. No completeness correction has been applied. 


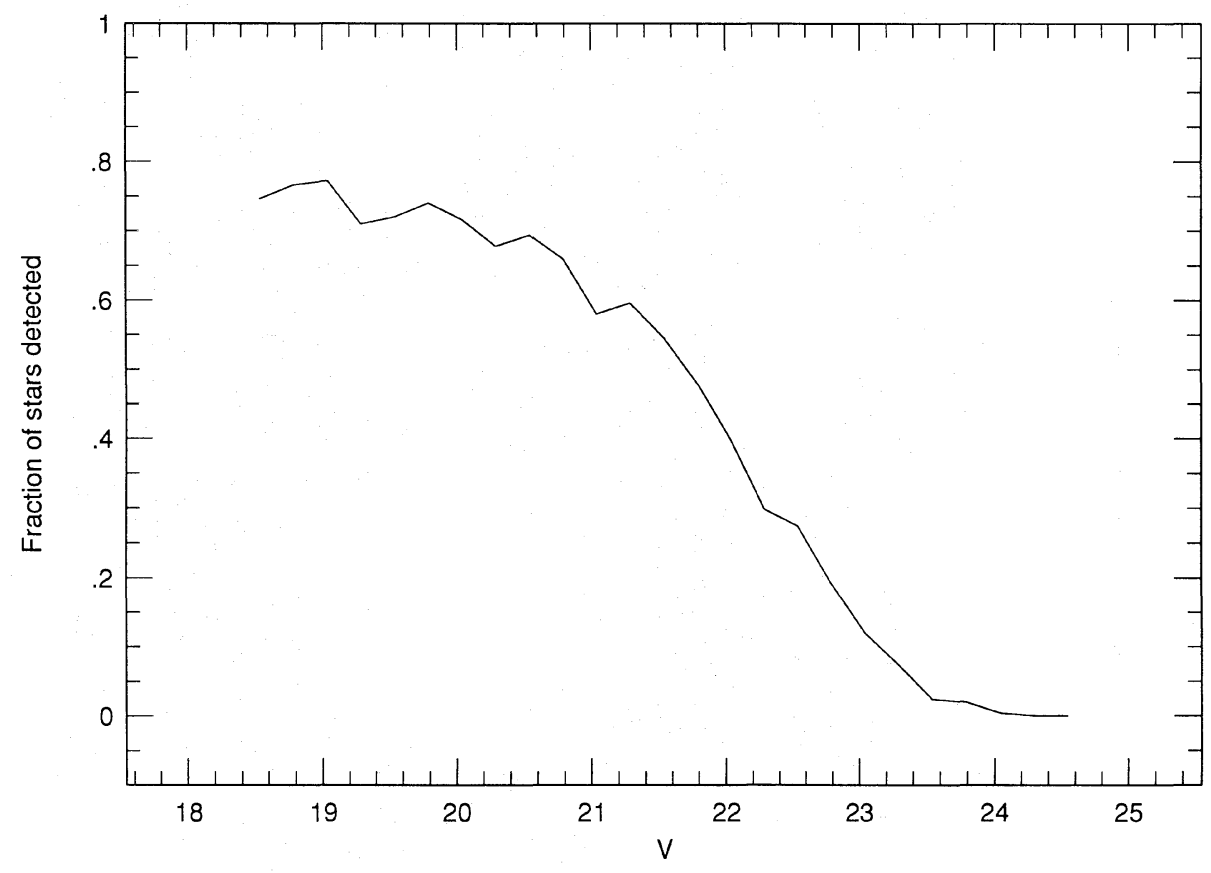

FIG. 4. Completeness estimates from simulated stars for WF2.

frame in bins of width 0.25 mag. The error bars here are from counting statistics only. No completeness correction or subtraction of foreground stars has been applied.

\subsection{Completeness and Error Estimates}

To determine a luminosity function for the bulge, accurate estimates of completeness and possible systematic photometric errors are required. Our approach to determining these was to place simulated stars of known magnitude on the frame, then attempt to find them and measure their brightness.

The PSFs used to reduce the frames were used to place artificial stars at random locations on each frame. Artificial stars were created with total counts ranging from approximately 40000 down to 150 , in steps of 0.25 mag. Stars were added at random locations on the frames, avoiding areas near the edge; the same star was added into both the F555W and the F785LP frame. We chose an average color of $V-I=1.4$ for the artificial stars, appropriate for the faint stars in the bulge. In each magnitude bin, 500 stars were added and measured, one at a time, for a total of 11000 artificial stars. The reduction techniques were identical to those used on the original frame. To be deemed a detection, the star had to be found on both the F555W and F785LP frames, the same criterion used for the real stars. If the artificial star was detected, its brightness was also measured to give an estimate of any systematic errors in the photometry.

Figure 4 shows the completeness results. The incompleteness at the bright end is caused by the masking of part of the frame around the saturated pixels. Consequently, applying these completeness estimates to the uncorrected luminosity function will give the number of stars per area of the chip, computed to be approximately 1.6 square arcmin. Figure 4 shows that, after allowing for masked areas, we are nearly complete down to $V \sim 21$; we sample about $50 \%$ of the stars at $V \sim 22.1$, and are almost totally incomplete by $V \sim 23.5$. It is difficult to determine to what magnitude the completeness corrections will give accurate results, but judging from differences in the resultant luminosity function using slightly different photometric techniques, we believe that the corrected luminosity function can be trusted to $V \sim 22$, but perhaps not much fainter.

We also used the simulated star experiments to try to determine whether there are systematic errors in the measured photometry. We found that, in general, the recovered photometry of the simulated stars was close to the expected brightness, but the stars were always measured a little bit too bright. The offset grew larger at fainter magnitudes, reaching approximately $0.1 \mathrm{mag}$ at $V \sim 21$. We have chosen not to make any correction for this possible systematic error; it would not significantly change our interpretation of the corrected luminosity function. Also, it is important to note that the simulated star experiments give only an estimate of the expected error and the situation for the true stars could be different, because in the simulated experiments the same PSF is used to create the star as to measure its brightness, whereas in the real situation, the PSF representation is not perfect.

\subsection{Corrected Luminosity Function}

We constructed a final luminosity function by applying the completeness correction to the raw counts. The $V$-band luminosity function is shown as the bold line in Fig. 5 . We have normalized the ordinate to stars per square arcmin per unit magnitude. The error bars in Fig. 5 come from 


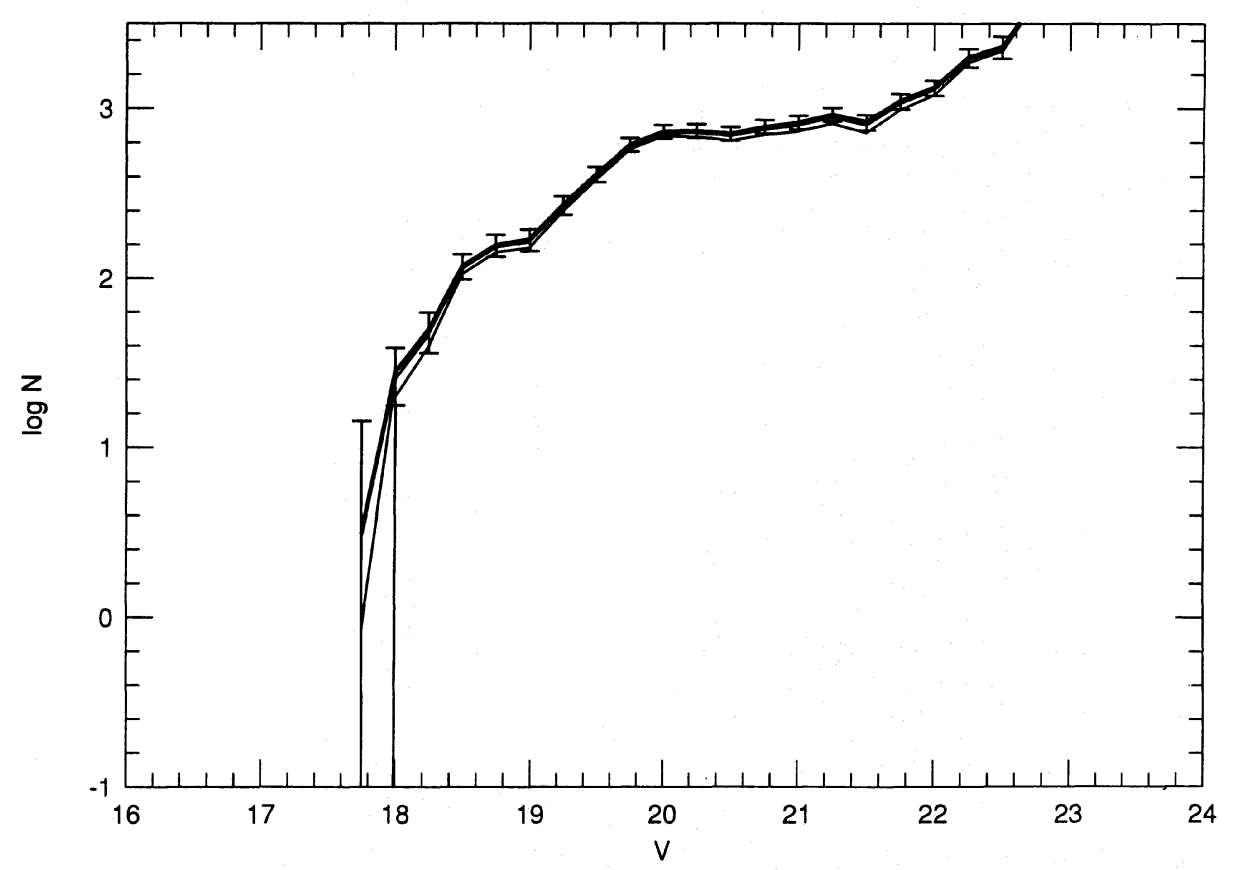

FIG. 5. The completeness-corrected luminosity function (bold line). Other lines represent luminosity function after foreground subtraction using the standard Bahcall-Soneira model and an adjusted model with three times as many disk stars.

counting statistics, including Poisson errors in both the observed star counts and in the artificial star counts used to determine the completeness correction.

\subsection{Foreground Contamination}

Since the line of sight to Baade's Window passes through the disk of the Galaxy, some foreground contamination of the luminosity function is expected. Terndrup (1988) determined a foreground correction by looking at the number of bright blue stars, assuming that these were foreground main-sequence stars. He used the BahcallSoneira (1980) model of the Galaxy to determine the expected number of foreground disk stars, and found that the model appeared to be deficient by a factor of about three. This is not a surprise since the model was not intended to be accurate for low Galactic latitudes. He adjusted the model by increasing the number of stars in several zones between the Sun and the Galactic center until the number of bright blue stars matched. The adjusted model was then used to subtract foreground disk stars over the entire observed range of magnitudes.

We cannot determine an independent foreground contamination estimate in the same way because all of the bright blue foreground stars are saturated on the WFC frames. Consequently, we have chosen simply to subtract the number of stars estimated by both the standard and an adjusted (Bahcall-Soneira) Galactic model; the adjusted model has three times as many disk stars. Even so, the number of bulge stars overwhelms the number of expected foreground stars; the foreground stars make up about 10 percent of the total number of stars at $V=18$, but only about 2 percent at $V=20$. Consequently, we find that the resultant shape of the luminosity function of the bulge is reasonably insensitive to the exact foreground subtraction. This is seen in Fig. 5, which (in addition to the bold line) shows the two foreground-corrected luminosity functions in the $V$ band. Subtracting a larger disk contribution would tend to steepen the slope of the the bulge luminosity function in the range $18<V<20$. However, it does not change the location of the break around $V=20$ in the luminosity function. We made separate foreground corrections for each of $V$ and $I$.

\section{DISCUSSION}

We wish to determine the properties of the bulge stars, in particular their typical metallicities and ages. This is complicated by the fact that there is almost certainly a wide range of metallicities present, and possibly some age spread as well. We also want to infer the slope of the initial mass function from the observed luminosity function of the unevolved stars.

We have three observed pieces of information, the color-magnitude diagram and the luminosity functions in each color. By comparison with stellar models, we can infer population characteristics. To do so, however, we must know the distance, reddening, and extinction in the direction of the field. The physical extent of the bulge also influences the observations, producing additional scatter in the color-magnitude diagrams and smearing of the intrinsic luminosity function.

In principle, it is possible to extract all of the unknowns 
(metallicity, age, reddening, extinction, and distance) from our observations alone, using differences in the shapes of the isochrones to determine the stellar properties. Once the correct isochrone is chosen, the amount of shifting in apparent magnitude and color required to match the model to the data tells us the distance and the reddening.

In practice, this is difficult because: (1) the population is not of a single metallicity and age, (2) the differences in isochrone shapes for different populations are not large in comparison with observational errors and uncertainties in the models, and (3) we sample only a limited region of the color-magnitude diagram in the bulge (basically the main sequence up to the turnoff).

Our approach here has been to first adopt parameters which have been independently determined for Baade's Window, and then to infer population characteristics. We discuss our adopted reddening and extinction in Sec. 4.1, our adopted distance and stellar distribution in Sec. 4.2, and our choice of stellar models in Sec. 4.3. Section 4.4 presents the model comparisons to the data using these inputs, and discusses how the inferred population characteristics change if different assumptions are made about the various inputs.

\subsection{Reddening and Extinction}

Estimates of the reddening and extinction towards Baade's Window have been discussed by a number of authors, including Kron \& Mayall (1960), Arp (1965), van den Bergh (1971), Zinn (1980), Frogel et al. (1984), Blanco et al. (1984), Walker \& Mack (1986), Rich (1988), and Terndrup (1988). Values of $\mathrm{E}(B-V)$ range from 0.46 to 0.59 for early-type stars. These reddening values have been determined from RR Lyraes near the globular cluster NGC 6522, from the giant branch of the cluster, and from some foreground stars in the direction of Baade's Window (which are still far enough away to be well out of the Galactic plane). Generally the lower estimates come from the foreground stars, so one might prefer the larger values.

Evidence for a variable extinction in Baade's Window comes from Blanco et al. (1984), who split Baade's Window up into three separate sections of differing extinction. They found absorption differences of several tenths of a magnitude between the different sections. Our field lies in the clearest part of their clearest section, so it is possible that the extinction is lower than the average extinction.

We have chosen to adopt a value of $\mathrm{E}(B-V)=0.46$ for our field, with the realization that this is uncertain. We computed the expected extinction in our bandpasses by using an atlas of stellar spectra, the extinction curve of Cardelli et al. (1989), the filter transmission curves, and the CCD QE curves. We find that the extinction for a $\mathrm{K} 0$ star is $A(\mathrm{~F} 555 \mathrm{~W})=1.38$ and $A(\mathrm{~F} 785 \mathrm{LP})=0.70$ using $R_{V}[\equiv A(V) / \mathrm{E}(B-V)]=3.0 ;$ for $R_{V}=3.3$, we obtain $A(\mathrm{~F} 555 \mathrm{~W})=1.50$ and $A(\mathrm{~F} 785 \mathrm{LP})=0.79$. Note that $\mathrm{E}(\mathrm{F} 555 \mathrm{~W}-\mathrm{F} 785 \mathrm{LP})$ is relatively insensitive to the adopted value of $R_{V}$; an increase in $R_{V}$ increases the extinction in both passbands equally. An increase in the amount of intervening dust would increase the extinction as well as making the stars redder. As a starting value, we adopt the values for a low $R_{V}$, because our data are consistent with isochrones only for a low extinction, as discussed further below.

\subsection{Distance to the Bulge and Physical Models}

The mean distance of the bulge stars should be similar to that of the Galactic center. Recent estimates of the distance to the Galactic center range from $7.1 \mathrm{kpc}$ (Reid 1989 ) to $8.4 \mathrm{kpc}$ (Rodgers et al. 1986). We adopt a distance of $8 \mathrm{kpc}$, which gives a true distance modulus of 14.52. Changing the distance by $1 \mathrm{kpc}$ would change the distance modulus by 0.25 to $0.3 \mathrm{mag}$.

The Galactic bulge extends over a range of Galactocentric radii. Various authors have discussed the physical structure of the bulge, and in particular whether the distribution of bulge stars is the same as the distribution of spheroid stars. To compare models with our observations of the luminosity function, some model of the spatial distribution needs to be adopted. A pure luminosity function from a set of stellar models will be smeared when stars at a variety of distances are sampled.

We have chosen to model the bulge distribution using the spatial distribution of the spheroid as described by the Bahcall-Soneira (1980) model. We have incorporated our model isochrones and luminosity functions into this model, and use it simply to integrate over distance. In its original form, the model predicts far fewer spheroid stars than are observed in our field. Several authors (e.g., Bahcall et al. 1983) have noted that an additional bulge component needs to be added to match star counts at low latitudes. We hope to address this issue in a future paper. For the present work, we have simply used the structural parameters of the Bahcall-Soneira spheroid to model the bulge distribution, but have arbitrarily increased the normalization to match the number of stars that we observe.

\subsection{Model Isochrones and Luminosity Functions}

Worthey (1993) has constructed an amalgamation of the isochrones of Vandenberg and collaborators (Vandenberg 1985; Vandenberg \& Bell 1985; Vandenberg \& Laskaraides 1987) and the Revised Yale Isochrones (Green et al. 1987), paying careful attention to the color transformations from the models to observed magnitudes. These isochrones provide data from low mass mainsequence stars up through the giant branch, and are available for a range of metallicities and ages.

A qualitative description of the behavior of the isochrones as a function of age and metallicity is useful. For older populations, the turnoff occurs at fainter magnitudes. Consequently, the break in the luminosity function occurs at a fainter magnitude, and the turnoff in the colormagnitude diagram moves down and to the right. A similar trend in both the color-magnitude diagram and the luminosity function is seen as the metallicity of the population is increased. These trends are demonstrated in Fig. 6. The top panel shows isochrones at two metallicities, 

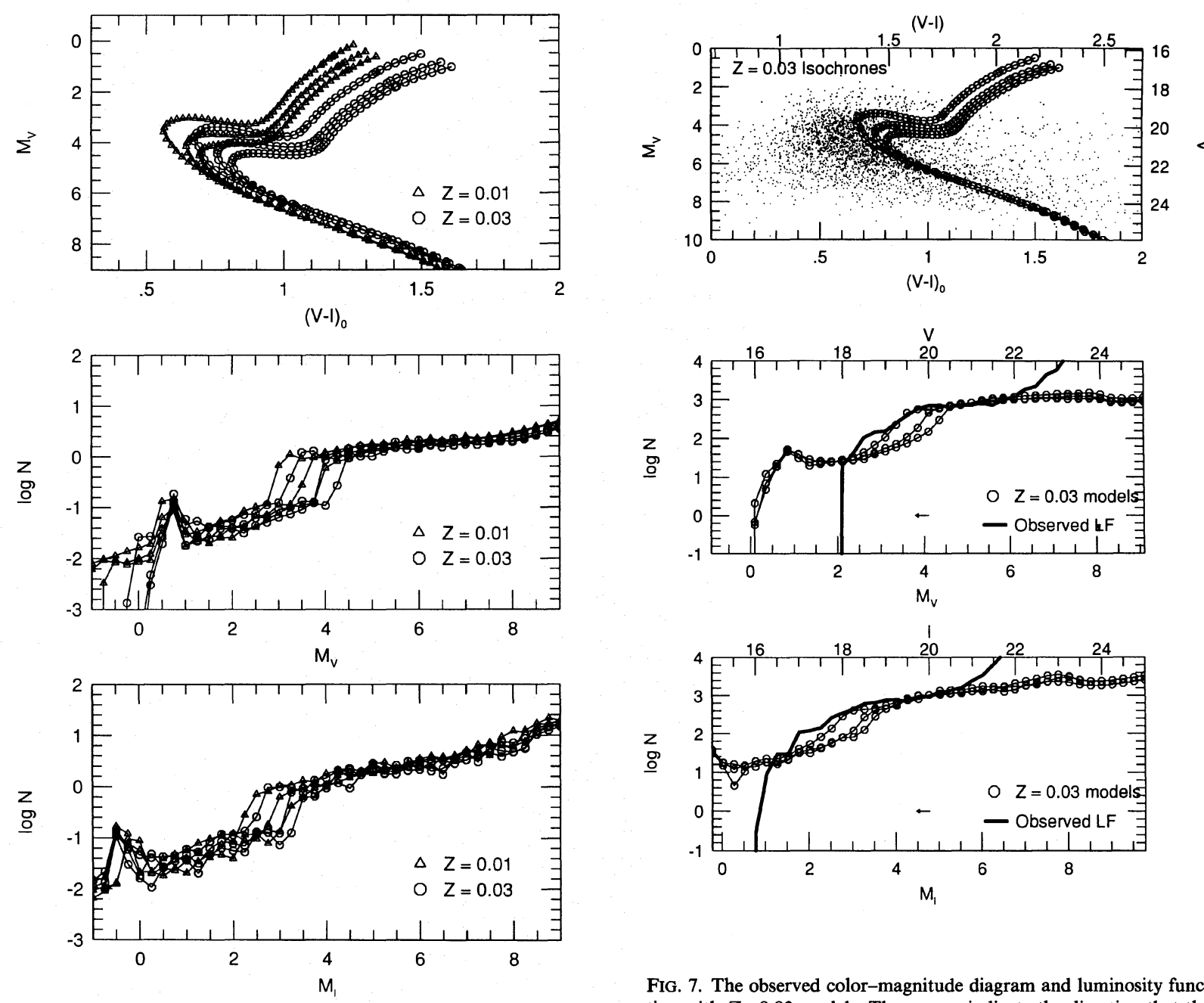

FIG. 7. The observed color-magnitude diagram and luminosity function with $Z=0.03$ models. The arrows indicate the direction that the model curves would move if the extinction in $V$ were lower by 0.3 mag.

FIG. 6. Model isochrones for two metallicities, $Z=0.01$ (triangles) and $Z=0.03$ (circles), for three different ages of 5,10 , and 15 billion years.

$Z=0.01$ (triangles) and $Z=0.03$ (circles), for the three different ages of 5,10 , and 15 billion years. The lower panels show luminosity functions for the same models.

\subsection{The Stellar Population of the Bulge}

We show the observed, foreground-subtracted (using the adjusted Bahcall-Soneira disk subtraction) luminosity function in $V$ and $I$ in the lower panels of Fig. 7 and a color-magnitude diagram in the upper panel. We have not "decontaminated" our color-magnitude diagram for foreground stars. The lower and left axes on the colormagnitude diagram represent the magnitudes after correction for our nominal values of the extinction, reddening, and distance to the bulge. The observed values are shown on the top and right. Similarly, on the luminosity function diagram, absolute magnitudes are on the bottom scale, and apparent magnitudes on top.

On Fig. 7, we plot the isochrones and luminosity func-

tions from the models with $Z=0.03$ for ages of 5,10 , and 15 billion years, with the older models having the break in the luminosity function at fainter magnitudes. The model luminosity functions have been normalized to match the observed number of stars at $V=21$ and $I=20$. For the color-magnitude diagrams, the models are plotted for a single distance; there is spread in the data from the range of distances sampled, from observational error, and from age and metallicity spread. In the luminosity functions, the models have been integrated over distance using the Bahcall-Soneira halo population distribution; the distance integration smears out the model luminosity function. If the bulge stars were more centrally concentrated than halo stars, the model luminosity functions would steepen around the turnoff. No spread of ages or metallicites has been included in the models. Figure 8 shows the same plots with $Z=0.01$ models. The model luminosity functions in these plots were constructed using a Salpeter initial mass function, with $d N / d M \propto M^{-2.35}$.

Since there is an observed spread in metallicity in the bulge, it is not appropriate to match models with a single 

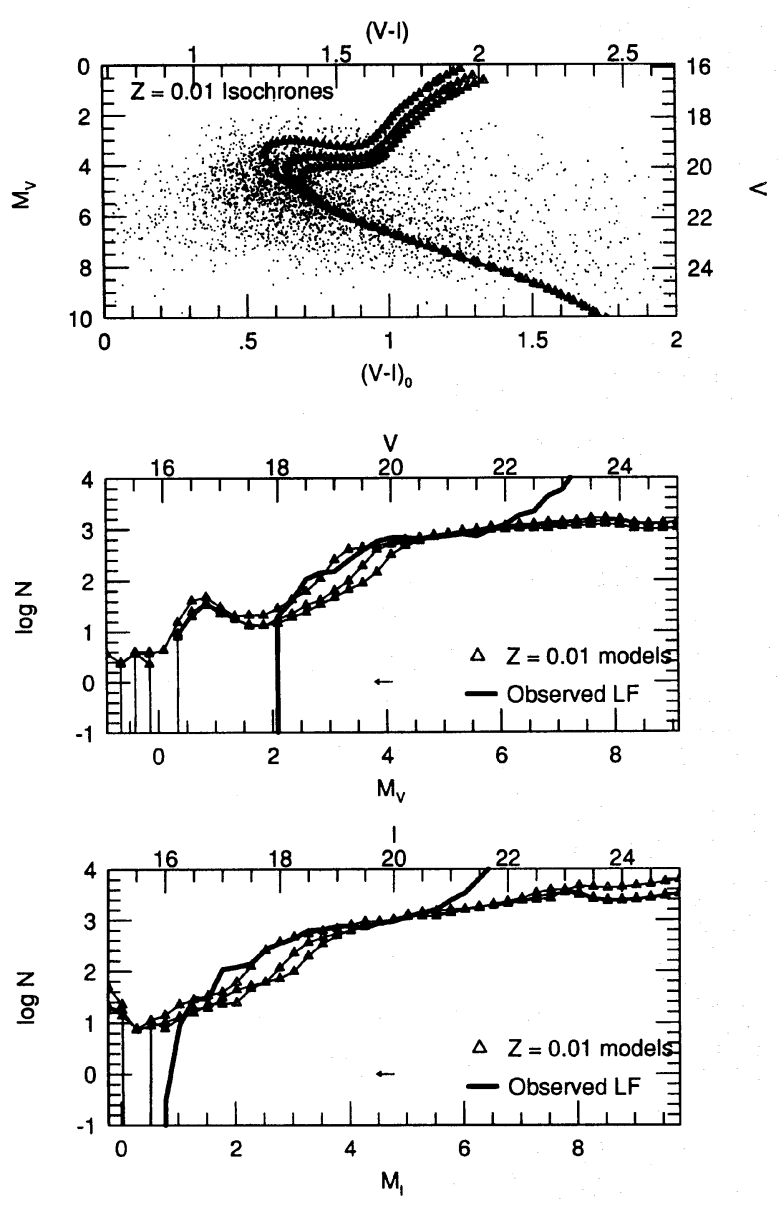

FIG. 8. The observed color-magnitude diagram and luminosity function with $Z=0.01$ models. The arrows indicate the direction that the model curves would move if the extinction in $V$ were lower by 0.3 mag.

metallicity. The mean metallicity of the bulge, based on the work of Rich (1988) and Geisler \& Friel (1992), is nearly twice solar, approximately corresponding to the $Z=0.03$ models. However, the metallicity distribution is asymmetric (see, e.g., Rich 1992), with metallicities as low as [Fe/ $\mathrm{H}] \sim-1.0$; the tail towards lower metallicities is longer than that towards higher ones. For older ages, the model luminosity functions are not strongly sensitive to metallicity changes; for younger populations, increasing the metallicity causes changes in the luminosity function similar to decreasing the age.

From the luminosity functions, it appears that an age of around $5 \mathrm{Gyr}$ for the bulge population provides a good fit regardless of the adopted metallicity with the nominal distance and reddenning. For a predominantly metal-rich population, however, the color-magnitude diagram is inconsistent with the data for any adopted age if our zero points are correct. For a metal-poor population, both the luminosity function and the color-magnitude diagram are consistent with an intermediate age population of between 5 and 10 Gyr. A mix of metallicites in the bulge would provide a locus of points in between the $Z=0.01$ and
$Z=0.03$ isochrones and thus would probably be consistent with the data, but the inferred age from the luminosity functions would still be less than $10 \mathrm{Gyr}$.

Because of zero-point uncertainties, conclusions based on the luminosity functions are more secure than those based on the color-magnitude diagram since errors in $V-I$ may be as large as 0.1 to 0.15 mag.

Note that the color-magnitude diagram includes some foreground stars. Judging from the Bahcall-Soneira model, however, we expect that the diagram is strongly dominated by bulge stars for $V \gtrsim 20$; however, the foreground stars may be preferentially bluer than the bulge stars if the bulge stars are more metal rich, so one might wish to slightly bias the observed points towards the red if one wishes to consider only bulge stars. This effect still would not make the color-magnitude consistent with the metal-rich isochrones.

The observed luminosity function is slightly shallower than ony of the models. This could be caused by a larger bulge width than we have included in our models, by an underestimate of the contamination by foreground stars at the bright end, or by random photometric errors which would smear the distribution.

\subsubsection{The effect of lower reddening}

If there is any significant component of the bulge that has greater than solar metallicity, the reddening must be lower than our adopted value if our zero points are accurate. To get a reasonable match to the isochrones at $Z=0.03$ would require $E(F 555 W-F 785 L P)$ to be reduced by about $0.15 \mathrm{mag}$, a significant decrease from our nominal value of 0.68 . We plot the color-magnitude diagram and luminosity functions for a reddening reduced by this amount in Figs. 9 and 10. The arrows in Figs. 7-10 show the amount the model curves would be moved for a reduction in $A(V)$ of $0.3 \mathrm{mag}$. With lower reddening and extinction, the luminosity functions still imply ages of less than 10 Gyr for both metallicities, although for the lower metallicity, the isochrones appear inconsistent with the color-magnitude diagram for any age.

To increase the inferred age to $10 \mathrm{Gyr}$ or larger would require the extinction to be lower by an additional few tenths of a magnitude; this would imply that the extinction in our field is only about half the measured extinction in other clear parts of Baade's Window, which, although not impossible, seems unlikely. The fact that the $I$-band luminosity function requires an age at least as young as the $V$-band luminosity function implies that a lower reddening will not be consistent with all of the current data. Also, with a very low extinction, the data would only be consistent for a metal-rich population.

\subsubsection{The effect of a shorter distance to the bulge}

The inferred age would be larger if the distance to the bulge were smaller than our adopted value of $8 \mathrm{kpc}$; if the bulge were at a distance of $7 \mathrm{kpc}$, the luminosity functions would move towards fainter magnitudes by 0.29 mag. For the nominal extinction, this change would still imply an 

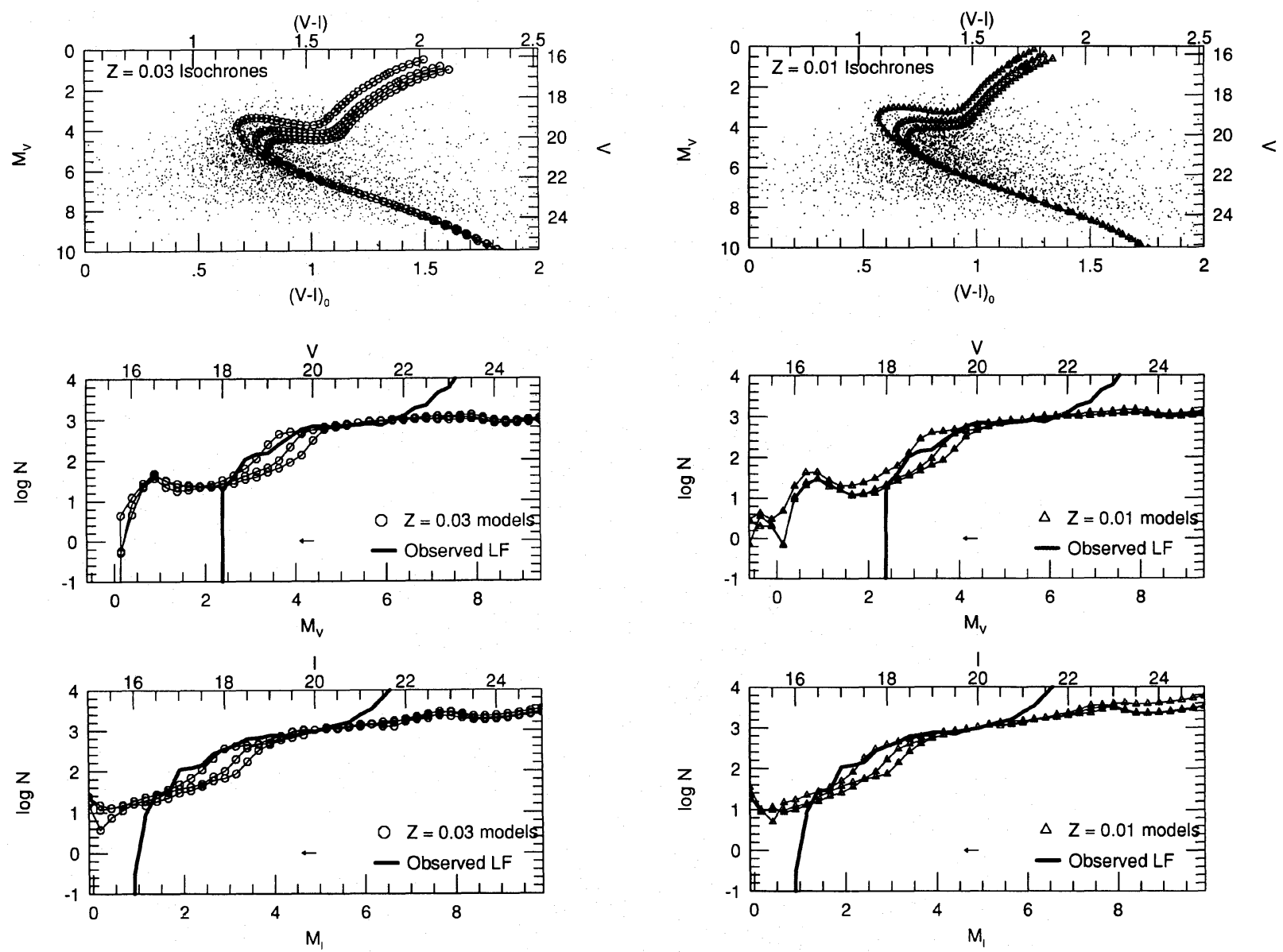

FIG. 9. Same as Fig. 7, except for a lower reddening.

FIG. 10. Same as Fig. 8, except for a lower reddening.

age of less than $10 \mathrm{Gyr}$. With a combination of a shorter distance to the bulge and a lower extinction, an age of 10 Gyr or greater would be inferred. A shorter distance would affect the $V$ - and $I$-band luminosity functions equally, and thus would be more consistent with the data than a very low extinction.

\subsubsection{The effect of systematic photometric errors}

Unfortunately, we must also consider the possibility that errors in the photometric zero points influence our results. A zero-point error of $0.1 \mathrm{mag}$ would change the results by a noticeable amount, but an age of less than 10 Gyr would still be inferred from the luminosity functions; conclusions based on the color-magnitude diagram are less secure. We have estimated our systematic errors to be less than $0.1 \mathrm{mag}$, but we recognize that accurate zero points are difficult to determine with an aberrated HST. The Lowell NGC 188 measurements imply that our $V$-and $I$-band measurements are too bright by 0.03 and 0.06 mag, respectively. Applying these adjustments would increase the inferred age, but not dramatically; they would make the $I$-band luminosity function more consistent with the $V$ band. If the simulated star experiments are correct for estimating systematic errors, the magnitudes for the stars

around the turnoff are too bright by a few hundredths of a magnitude; this would also drive the inferred age towards a larger value.

We have obtained some ground-based magnitudes in our Baade's Window field (Mateo 1993) for a few stars which are among the least crowded in the field; of these stars, several have ground and HST magnitudes within 0.1 mag of each other, although a few others appear significantly (several tenths) fainter from the ground. Given the severe crowding from the ground, it is difficult to justify adopting the ground magnitudes as truth; mostly, we were convinced from looking at the ground-based pictures of how much HST can add to knowledge of the stellar population of the bulge!

Our zero points are more uncertain than we would like, and consequently so is the interpretation. We look forward to more observations with a better calibrated system and a sharper PSF to confirm our magnitudes.

\subsubsection{Summary}

Using the nominal extinction and distance to the bulge, our data imply an intermediate age for low-metallicity isochrones $(Z=0.01)$ and are inconsistent with any isochrones at high metallicity $(Z=0.03)$ if our photometric 


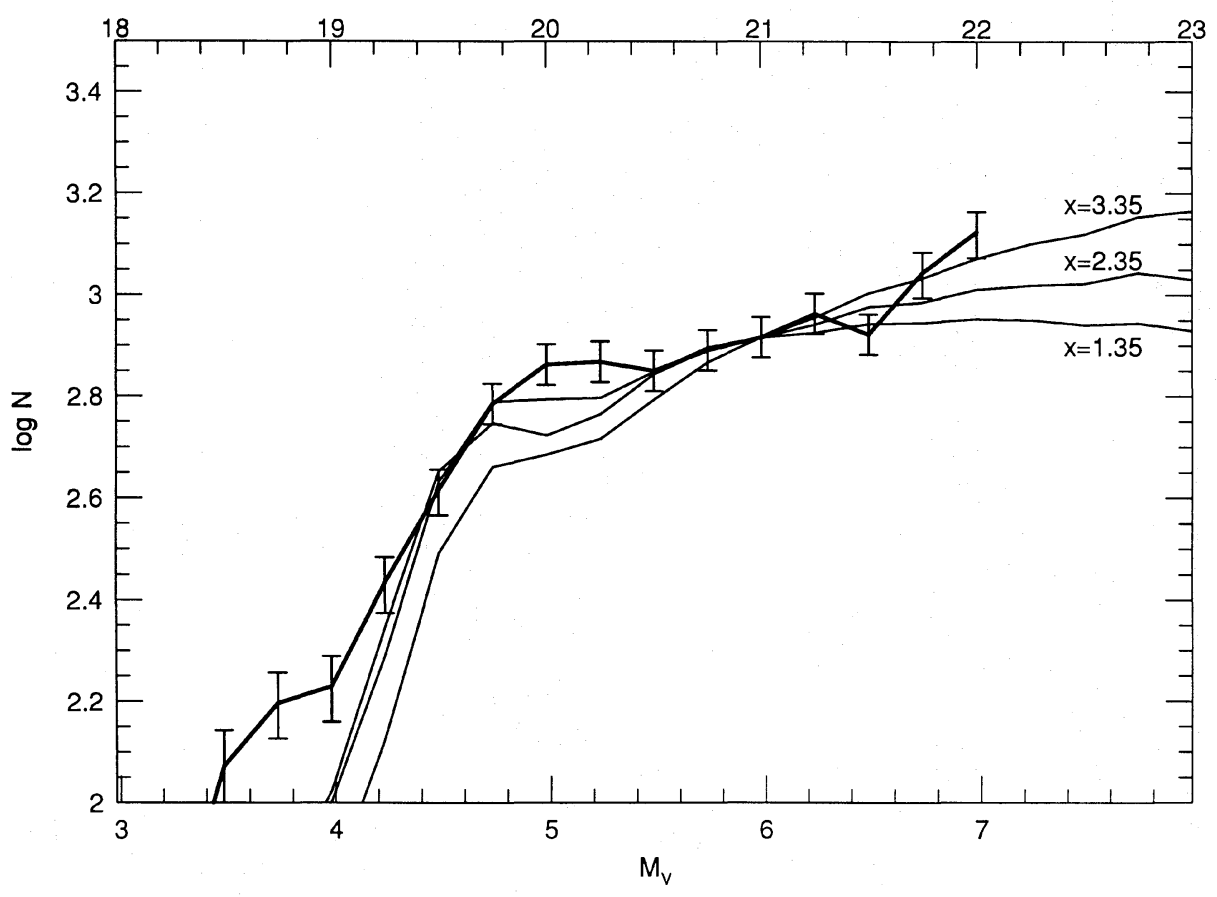

FIG. 11. The observed luminosity function along with predictions from the Bahcall-Soneira model using model luminosity functions assuming IMFs with three different slopes, $x=1.35,2.35,3.35$. The flattest curve corresponds to $x=1.35$, the steepest to $x=3.35$.

zero points are correct. A mix of metallicities is probably consistent with the data, but still only for an intermediate age. With a lower reddening, the data can be made consistent for the higher metallicity, but the inferred age is still less than $10 \mathrm{Gyr}$. A shorter distance to the bulge would increase the inferred age, but the lower reddening is still required to match the color-magnitude diagram for high metallicity; a combination of a short distance and low metallicity would imply an age of $10 \mathrm{Gyr}$ or greater. These conclusions depend on our ability to determine fairly accurate zero points, and should be confirmed with a better calibrated system.

\subsection{The Main-Sequence Luminosity Function and the IMF}

Over the range $20<V<22$, we can measure the luminosity function of the bulge main-sequence stars with reasonable accuracy. This corresponds to a mass range from approximately 0.8 to 1.1 solar masses. The model luminosity functions in Figs. 7 and 8 were computed assuming a Salpeter IMF with slope -2.35 . Since these appear to match the observed luminosity function moderately well, we infer that the bulge IMF in this mass range does not significantly differ from a "standard" IMF. We also note that when we use the Bahcall-Soneira model, with an observed solar neighborhood luminosity function, the computed luminosity function is also consistent with our observed function for the bulge.

The completeness estimates are uncertain fainter than $V \sim 22$, so the apparent upturn of the luminosity function at the faintest magnitudes is questionable. We found that formal fits to the IMF slope were sensitive to the magnitude cutoff that we imposed on the observed points, because the faintest points show an upturn which may not be real, but which implies large IMF slopes.

Since the bulge stars are located at a range of distances, the observed IMF will be smeared, so the slope that we infer from assuming a single distance may underestimate the true IMF slope by an amount depending on the structure of the bulge. We incorporated IMFs with different slopes into the Bahcall-Soneira model to try to account for this smearing. Figure 11 shows three model luminosity functions with slopes of $x=1.35,2.35,3.35$, along with the observed data; the models are for a $5 \mathrm{Gyr}$ population with $Z=0.03$. The model curves have been arbitrarily scaled to a fixed value at $V=21$, so one is free to shift these curves vertically to find a good match. A lower extinction would shift the curves to the left.

The curve with the shallowest slope is the $x=1.35$ case. It appears to be most consistent with the data at the bright end, but a steeper slope better fits the data at the faint end; the IMF slope is not well constrained. Given the uncertainties, the slope is consistent with either a Salpeter IMF or a solar neighborhood luminosity function. We eagerly await data at fainter magnitudes (to be obtained with WFPC-2) to better constrain the slope of the IMF.

\section{CONCLUSIONS}

We have observed a field in Baade's Window with the WFC, and we obtain $V$ - and $I$-band photometry. By add- 
ing artificial stars, we determine completeness corrections and believe that we can measure a luminosity function down to $V \sim 22$. The location of the break in the luminosity function suggests that there may be a significant number of intermediate age stars in the Galactic bulge. Both the color-magnitude diagram and the observed luminosity functions can be matched well if the reddening in our field is slightly less than the reddening in the rest of Baade's Window and if the bulge contains a significant population of stars of age 5-10 Gyr with a range of metallicities. It would take a conspiracy of lower reddening, a shorter distance to the bulge, and/or photometric zero-point errors to make our data consistent with an exclusively old population in the bulge. Adjustments in any of these parameters would drive the inferred age towards $10 \mathrm{Gyr}$. The accuracy of our photometry should be confirmed with a better calibrated system.

If the bulge has a substantial component of stars of age $<10 \mathrm{Gyr}$, this will cause us to reconsider formation scenarios for the Galaxy which have the bulge forming at epochs comparable with the formation epoch for the globular clusters. If the intermediate age is confirmed, it will be of great interest to try to get a more accurate age estimate for the bulge to determine if it is older than the oldest disk stars; it would be challenging to understand how a dense stellar system might form in the center of the Galaxy after the disk of the Galaxy was formed.

We measure the luminosity function in the bulge between $20<V<22$, but the IMF slope is poorly constrained because we sample only a narrow range of stellar mass and because our luminosity function is uncertain at the faintest magnitudes. The data are consistent with the local solar neighborhood slope.

We would like to thank Mario Mateo and collaborators for sharing with us their ground-based pictures and magnitudes of stars in Baade's Window. This research was conducted by the WFPC Investigation Definition Team, supported in part by NASA Grant No. NAS5-1661.

\section{REFERENCES}

Arp, H. 1965, ApJ, 141, 43

Baade, W. A. 1946, PASP, 58, 249

Baade, W. A. 1951, Publ. Univ. Mich. Obs., 10, 7

Bahcall, J. N., \& Soneira, R. M. 1980, ApJS, 44, 73

Bahcall, J. N., Schmidt, M., \& Soneira, R. M. 1983, ApJ, 265, 747

Baum, W. A., Light, R. M., Holtzman, J. A., Hunter, D. A., Kreidl, T. J., O'Neil, E. J., \& Groth, E. J. 1992, Galactic Bulges, IAU Symposium No. 153, edited by H. Habing and H. Dejonghe (Kluwer, Dordrecht) (in press)

Blanco, V. M., McCarthy, M. F., \& Blanco, B. M. 1984, AJ, 89, 636

Burrows, C. J., Holtzman, J. A., Faber, S. M., Bely, P. Y., Hasan, H., Lynds, C. R., \& Schroeder, D. 1991, ApJ, 369, L21

Caputo, F., Chioffi, A., Castellani, V., Collados, M., Roger, C. M., \& Paez, E. 1990, AJ, 99, 261

Cardelli, J. A., Clayton, G. C., \& Mathis, J. S. 1989, ApJ, 345, 245

Cohen, J. G., Frogel, J. A., Persson, S. E., \& Elias, J. H. 1981, ApJ, 249, 481

Frogel, J. A., Whitford, A. E., \& Rich, R. M. 1984, AJ, 89, 1536

Geisler, D., \& Friel, E. D. 1992, AJ, 104, 128

Green, E. M., Demarque, C. P., \& King, C. R. 1987, Revised Yale Isochrones and Luminosity Functions (Yale University Press, New Haven) Harris, H. C., Baum, W. A., Hunter, D. A., \& Kreidl, T. J. 1991, AJ, 101, 677

Holtzman, J. A. 1989, PASP, 102, 806
Kron, G. E., \& Mayall, N. J. 1960, AJ, 65, 581

Lauer, T. R. 1989, PASP, 101, 445

Lee, Y. W. 1993, AJ (in press)

Mateo, M. 1993, private communication

Olson, B. I. 1975, PASP, 87, 349

Reid, M. J. 1989, in The Center of the Galaxy, IAU Symposium No. 136, edited by M. Morris (Kluwer, Dordrecht), p. 37

Rich, R. M. 1988, AJ, 95, 828

Rich, R. M. 1992, in The Center, Bulge, and Disk of the Milky Way, edited by L. Blitz (Kluwer, Dordrecht), p. 47

Rodgers, A. W., Harding, P., \& Ryan, S. 1986, AJ, 92, 600

Stetson, P. B. 1987, PASP, 99, 191

Terndrup, D. M. 1988, AJ, 96, 884

Terndrup, D. M., Frogel, J. A., \& Whitford, A. E. 1991, ApJ, 378, 742 van den Bergh, S. 1971, AJ, 76, 1082

Vandenberg, D. A. 1985 , ApJS, 58, 711

Vandenberg, D. A., \& Bell, R. A. 1985, ApJS, 58, 561

Vandenberg, D. A., \& Laskarides, P. G. 1987, ApJS, 64, 103

Walker, A. R., \& Mack, P. 1986, MNRAS, 220, 69

WFPC SV Report 1992, edited by S. M. Faber

Whitford, A. E., \& Rich, R. M. 1983, ApJ, 274, 723

Wood, P. R., \& Bessell, M. S. 1983, ApJ, 265, 748

Worthey, G. 1993, ApJ (in press)

Zinn, R. 1980, ApJS, 42, 19 